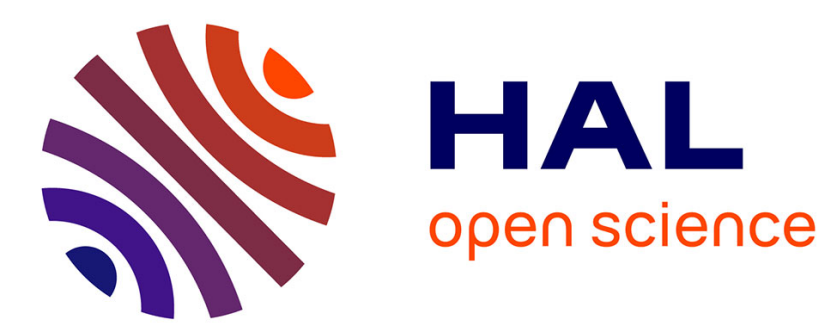

\title{
Narrow-band "noise" and damping in NbSe3
}

J. Richard, Jie Chen, S. Artemenko

\section{- To cite this version:}

J. Richard, Jie Chen, S. Artemenko. Narrow-band "noise" and damping in NbSe3. Journal de Physique IV Proceedings, 1993, 03 (C2), pp.C2-33-C2-38. 10.1051/jp4:1993206 . jpa-00251575

\section{HAL Id: jpa-00251575 https://hal.science/jpa-00251575}

Submitted on 1 Jan 1993

HAL is a multi-disciplinary open access archive for the deposit and dissemination of scientific research documents, whether they are published or not. The documents may come from teaching and research institutions in France or abroad, or from public or private research centers.
L'archive ouverte pluridisciplinaire HAL, est destinée au dépôt et à la diffusion de documents scientifiques de niveau recherche, publiés ou non, émanant des établissements d'enseignement et de recherche français ou étrangers, des laboratoires publics ou privés. 


\title{
Narrow-band "noise" and damping in $\mathrm{NbSe}_{3}$
}

\author{
J. RICHARD, J. CHEN and S.N. ARTEMENKO (1) \\ Centre de Recherches sur les Très Basses Températures, Laboratoire associé à l'Université Joseph Fourier, \\ CNRS, BP. 166, 38042 Grenoble cedex 9, France
}

\begin{abstract}
We report the results of a Narrow-band "Noise" (NBN) study, in $\mathrm{NbSe}_{3}$ for magnetic fields up to $19 \mathrm{~T}$ and temperatures as low as $15 \mathrm{~K}$. The first result of this NBN study is the observation of the decrease of $\mathrm{dJ}_{\mathrm{CDW}} / \mathrm{dv}$ with decreasing temperatures for both transitions. We report also new values for the absolute value dJCDW/ $d v$ for both transitions, and the existence of a curvature of $\mathrm{d} \mathrm{J}_{\mathrm{CDW}} / \mathrm{dv}$ versus frequency, in low frequency range. We have interpreted the decrease of $\mathrm{dJ} / \mathrm{CD} / \mathrm{dv}$ with temperatures in terms of Artemenko's theory which has been used in 1984 to explain the non-linear Hall effect in $\mathrm{TaS}_{3}$. The total longitudinal current is given by $\mathrm{J}_{\mathrm{T}}=\mathrm{JCDW}(1-\mathrm{A})+\sigma_{1} \mathrm{E}$, where the therm -AJCDW is a backward normal current flow due to the moving CDW. From the saturing value of $\mathrm{dE} / \mathrm{dv}$ obtained in high electric field we have deduced the total damping parameter $\tau^{-1}$ which is decreasing with temperature. Using data obtained under application of a magnetic field of $7 \mathrm{~T}$ and the Wonneberger's theory for the ac response of the collective mode, we have deduced the thermal variation of the bare phason damping coefficient $\tau_{\mathrm{o}}{ }^{-1}$. We report that for $\mathrm{T}<30 \mathrm{~K}, \tau_{\mathrm{o}}{ }^{-1} \sim \mathrm{AT}^{4.5}$ very close to the theoretical predictions of Takada, Wong and Holstein.
\end{abstract}

Among all low-dimensional conductors which are known to display "sliding" (CDW's) conduction, $\mathrm{NbSe}_{3}$ is certainly the most widely studied in terms of CDW dynamics [1]. $\mathrm{NbSe}_{3}$, which undergoes two CDW transitions $\left(\mathrm{T}_{1}=145 \mathrm{~K} ; \mathrm{T}_{2}=59 \mathrm{~K}\right)$, is the only one to remain semimetallic at all temperatures. A striking phenomenon in CDW systems is the presence of a radio frequency voltage [2], improperly referred to as narrow band "noise" (NBN), when the dc bias exceeds a threshold electric field $\mathrm{E}_{\mathrm{T}}$. The oscillations are the hallmark of the sliding conduction of the CDW, with a fundamental frequency $v_{0}$ shown to be proportional to the excess current density JCDW carried by the CDW. From the ratio $\mathrm{P}=\mathrm{J}$ CDW $/ v_{0}$ the number of condensed carriers can be inferred [3]. NBN has been studied extensively, but up to now no accurate measurements have been reported for low temperatures ( $T<90 \mathrm{~K}$ for the first transition, and $\mathrm{T}<25$ for the second transition) and frequencies higher than $30 \mathrm{MHz}$.

We report on extremely precise NBN experiments made over the range $65 \mathrm{~K}<\mathrm{T}<150 \mathrm{~K}$ for the first transition and $15 \mathrm{~K}<\mathrm{T}<55 \mathrm{~K}$ for the second transition.

Making NBN measurements at low temperature (compared with $T_{1}$ or $T_{2}$ ) we are faced with the problem of removing sample heating effects, due to the application of high DC electric fields in order to depin the CDW. To reduce sample Joule heating, we have performed four probe resistance measurements by the pulsed technique, with the sample immersed in a cryogenic liquid such as Argon or Nitrogen for the first transition, Neon or Hydrogen for the second transition. Using high quality samples with minimal threshold fields $(9 \mathrm{mV} / \mathrm{cm}$ at $48 \mathrm{~K}$ ) we were able for the first time to do Fourier analysis of the current oscillations for samples depinned by a pulsed current. With this technique, pulsed widths of $50 \mu$ s to $1 \mathrm{~ms}$ have been used with duty cycles of $10^{-4}$.

Most of the NBN studies in $\mathrm{NbSe}_{3}$ so far reveal a linear relation between JCDW and $\mathrm{v}_{0}$ over the usual frequency range $0-30 \mathrm{MHz}$. A few measurements have been made up to 400 
$\mathrm{MHz}$ but only at a few temperatures. $\mathrm{NbSe}_{3}$ samples which exhibit a non linear relation between JCDW and $v_{0}$ have been considered to be poor quality samples. Suprisingly, in orthorhombic or monoclinic $\mathrm{TaS}_{3}[5]$ and $\left(\mathrm{NbSe}_{4}\right)_{10 / 3} \mathrm{I}[6]$ curvature can always be found in some specific temperature range. In order to improve our sensitivity, we have plotted the experimental derivative $\mathrm{dJ}_{\mathrm{CDW}} / \mathrm{dvo}$ versus frequency $v_{\mathrm{o}}$. Our results shown in Fig. 1 indicate that even for high quality samples with coherent oscillations, non linearity is present at $45 \mathrm{~K}$ for $v_{0}<30 \mathrm{MHz}$. Unlike $\mathrm{TaS}_{3}$, the curvature is more prominent at lower temperatures for both transitions. We also have performed NBN measurements on ( $\left.\mathrm{TaSe}_{4}\right)_{2} \mathrm{I}$ and observed curvature, even at $160 \mathrm{~K}$, but in the frequency range $0-3 \mathrm{MHz}$. We argue, therefore, that the curvature exists for all samples exhibiting NBN, its amplitude is very temperature dependent, but for high enough frequency the linearity between JCDW and $v_{0}$ will be recovered. For $\mathrm{NbSe}_{3}$ almost all previous measurements made below $120 \mathrm{~K}$ and $25 \mathrm{~K}$ give an underestimate of the ratio $\mathrm{J}_{\mathrm{CDW}} / \mathrm{v}_{\mathrm{o}}$.

The linearity observed in previous measurements is often the consequence of a too small range of frequency (0-30 MHz), an example is given on the insert of Fig. 2.

Fig. 3 shows the result of a temperature dependent study for both transitions [4]. Each data point is actually the saturated value $P=d J_{C D W} / \mathrm{dv}_{\mathrm{o}}$ after correction of the heating effects. Studies have been carried out on more than 20 crystals and the overall behaviour is not sample dependent. $P_{1}$ (the average value on 14 samples) is equal to $40.2 \mathrm{~A} \mathrm{MHz}^{-1} \mathrm{~cm}^{-2}$ [8] and $0.75 \mathrm{P}_{1}<\mathrm{P}_{2}<0.85 \mathrm{P}_{1}$, depending of the sample. The previous results have been evaluated in the regime where $P_{1}$ or $P_{2}$ do not saturate which explains the scatter of the data published by various authors [1]. With the above value for $P_{1}$ we deduce for $\lambda$ pinning $/ \lambda C D W$ a value of 0.93 , this is very close to 1 , in agreement with the classical model, as already observed in $\mathrm{TaS}_{3}$ [7]. The central result of our study is the observation of the decrease of $\mathrm{dJ} C \mathrm{CW} / \mathrm{dv}_{\mathrm{o}}$ with temperature for both transitions, strongly at variance with various theories.

For the second transition we have performed NBN measurements under application of magnetic fields. In Fig. 4 we plot the saturated value $\mathrm{dJ} C D W / d v_{0}$, after correction of heating effects, versus temperature, at $B=0$ and $B=7 T$.

Magnetic field has only a small effect $(4-7 \%)$ on the slope $P_{2}$ for temperatures below $45 \mathrm{~K}$. For temperatures above $45 \mathrm{~K}$ any effect of the magnetic field is lost in our experimental resolution $(\sim 1 \%)$.

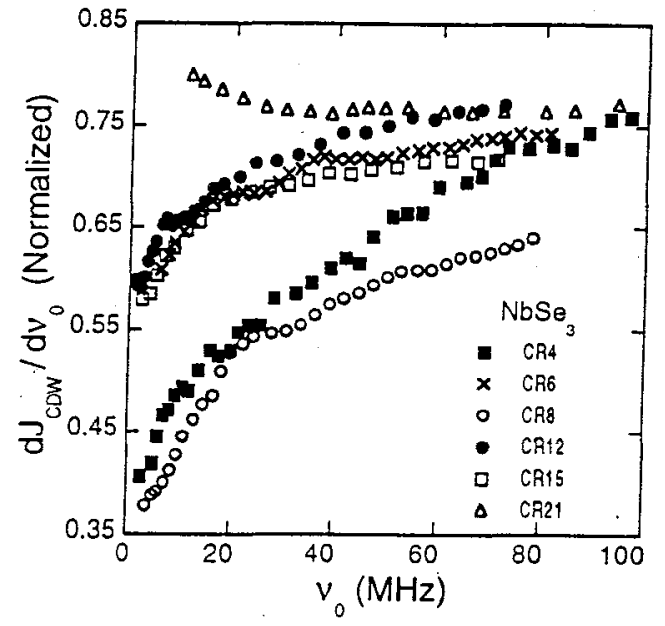

Figure 1

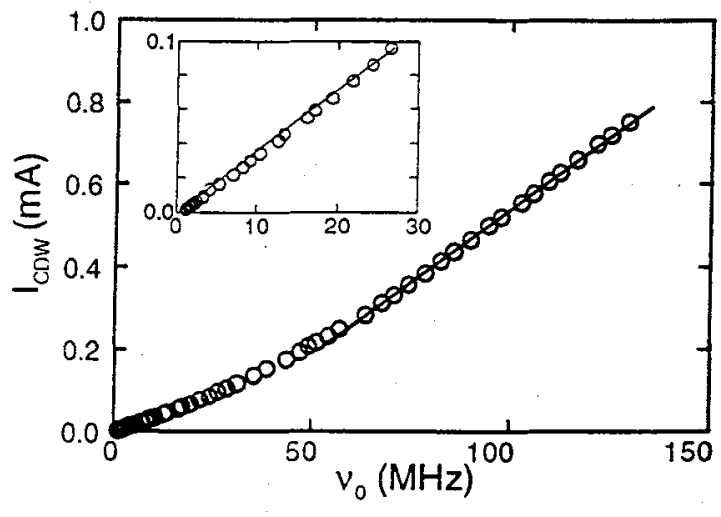

Figure 2

Figure 1 :Experimental slope $\mathrm{dJ} C D W / d v_{0}$ (normalized for each sample to the maximum value obtained at the first transition) versus frequency for different samples at $\mathrm{T}=45 \mathrm{~K}$.

Figure $2: I_{C D W}$ versus frequency at $\mathrm{T}=36 \mathrm{~K}$. The data show that the linearity obtained in low frequency range (see insert) is misleading, as shown by the measurements made in the higher frequency range. 
Concerning the interpretation of the decrease of $\mathrm{P}$ as $\mathrm{T}$ falls we favor the idea that the independent two fluid model is not strictly valid. Restrictions to this model have already been published by different authors (Boriak-Overhauser, Lee and Rice [9]). In 1984, Artemenko et al. explained the non linear Hall effect observed in $\mathrm{TaS}_{3}$ [10], by taking into account the effect of a moving CDW on the distribution of quasiparticles. For $\mathrm{NbSe}_{3}$ which is still metallic below the Peierls transitions, we must condider a slightly different model with electronic pockets. Unlike semiconducting Peierls materials the imperfect nesting parameter $\varepsilon_{o}$ is not very small compared to $\Delta$. Due to the 2D pockets we must now define $\delta_{1,2}(\delta<<\Delta)$, the energy difference between the Ferni level and the energy in the minimum and the maximum, respectively. To obtain the current, a linearized kinetic equation for a quasiparticle distribution function has been solved. With $\mathrm{T}<\Delta$ detailed calculations can be found for semiconducting Peierls compounds in Ref. 11. If we consider the simple symmetric case $\left(\delta_{1}=\delta_{2}\right)$, where there is no anisotropy in the effective mass, the total longitudinal current is given by :

$$
\mathrm{J}_{\mathrm{T}}=\mathrm{J}_{\mathrm{CDW}}(1-\mathrm{AF}(\mathrm{d}))+\sigma_{1} \mathrm{E},
$$

where

$$
A=\left(1+2 \alpha^{-1}\right)^{-1} \delta / \sqrt{\varepsilon_{0} \Delta} \text {, and } F(d)=\ln \mathrm{d}^{-1}\left(1+\mathrm{e}^{\mathrm{d}}\right)+(1+\alpha)^{-1} \text { th } \mathrm{d} / 2 \text {, }
$$

where $\alpha=v_{b} / v_{f}, \delta=\delta_{1}+\delta_{2}, d=\delta / 2 T, \sigma_{1}$ is the quasiparticle conductivity and $v_{b}$ and $v_{f}$ are backward and forward inverse scattering time respectively.

Due to the moving CDW a backward normal current flow, proportional to JCDW,

$\left(\mathrm{J}_{B}=-A F(d) J_{C D W}\right)$ is added to JCDW and to the contribution of the quasiparticules. The presence of $\mathrm{J}_{B}$ results in non-monotonous temperature dependence of that part of the current which is proportional to the CDW velocity and hence to the $N B N$ frequency. In $\mathrm{NbSe}_{3}, J_{B}$ can be large enough to be taken into account, unlike semiconducting Peierls materials where $J_{B}$ is exponentially small at $T<\Delta$.

The possibility of non-monotonous temperature dependence of $\mathrm{J}_{B}$ for semiconducting $\mathrm{CDW}$ material without electronic pockets for high temperature $\mathrm{T} \gtrsim \Delta$ was shown by Maki and Huang [12]. The non-monotonous behaviour of $\mathrm{J}_{\mathrm{B}}$, given by equation (1) is less pronounced than the experimentally observed one. A fit to equation (1) has not too much physical signification due to the fact that we neglect the mass anisotropy and temperature dependence of $\varepsilon_{0}, \delta$ and $\alpha$. In the regime when $T<\delta$ we can approximate the back flow current by :

$$
\mathrm{J}_{\mathrm{B}} / \mathrm{J} \mathrm{CDW}=-4 \mathrm{n} \varepsilon_{\mathrm{F}}\left(\alpha^{-1}+2^{-1}\right)^{-1} / \mathrm{N} \Delta
$$

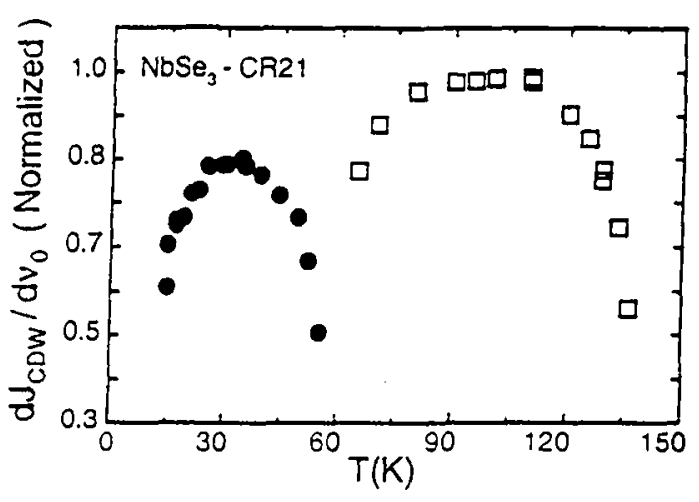

Figure 3

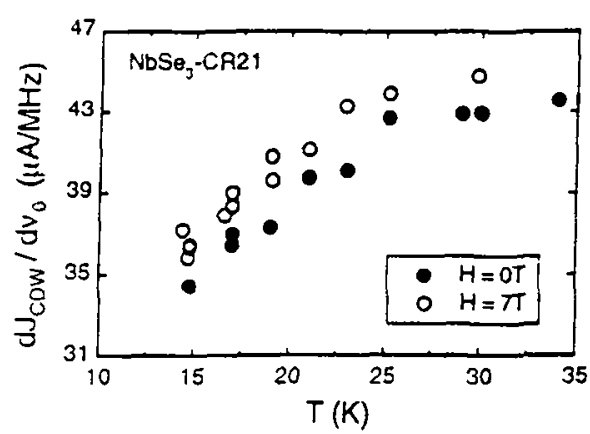

Figure 4

Figure 3 : Temperature dependence of $d J_{C D W} / d v_{0}$, for both transitions normalized to the maximum value of the first transition.

Figure 4 : Temperature depence of $d J_{C D W} d / v_{0}$ for the second transition with and without an applied magnetic field. 
where $\varepsilon_{F}$ is the Fermi energy, $n$ the number of quasiparticles and $N$ the total number of carriers for the second transition. Using the estimated parameters, $\Delta_{\mathrm{o}}=350 \mathrm{~K}, \varepsilon_{\mathrm{F}}=4200 \mathrm{~K}$, $\mathrm{N}=2 \times 10^{21}$ carriers $/ \mathrm{cm}^{3}$ and $\alpha=1$, We obtain the experimental extrapolation at $4 \mathrm{~K}$ $\left(\mathrm{J}_{\mathrm{B}} / \mathrm{JCDW}=-0.45\right)$ for $\mathrm{n}=1.9 \times 10^{19}$ which is in aggrement with Hall effect measurements $\left(\mathrm{ln}_{\mathrm{o}}-\mathrm{p}_{\mathrm{o}} \mathrm{l}=7 \times 10^{18}\right.$ carriers $\left./ \mathrm{cm}^{3}\right)$. The effect of the magnetic field on $\mathrm{dJ} \mathrm{CDW} / \mathrm{dv}_{\mathrm{o}}$ is interpreted in terms of a magnetoresistance effect on $J_{B}$ rather than an increase of $n$.

The interaction of the moving condensate with thermally ambient phonons or phasons and with the uncondensed electrons leads to an effective damping parameter $\tau^{-1}$ and a viscosity $\eta$, with $\eta \propto \tau^{-1}$. Fleming $e t$ al. [13] have observed an Arrhenius behavior of the excess $\mathrm{CDW}$ current I $\mathrm{CDW}$ in different materials $\left(\mathrm{K}_{0.3} \mathrm{MoO}_{3}, \mathrm{TaS}_{3},\left(\mathrm{TaSe}_{4}\right)_{2} \mathrm{I}\right)$. These results suggest that the zero-frequency viscosity of the CDW carriers diverges at low temperatures.

A classical starting point for a description of the CDW dynamics has been a phenomenological single-particle description.

For frequencies $v<500 \mathrm{MHz}$ the inertial term can be neglected compared to the damping term. The electric field is given by:

$$
\mathrm{E}=\mathrm{E}_{\mathrm{T}}+\eta \frac{\mathrm{V}_{\mathrm{CDW}}}{\mathrm{Ne}}
$$

where $\mathrm{E}_{\mathrm{T}}$ is the threshold electric field, $\mathrm{V}_{\mathrm{CDW}}$ the $\mathrm{CDW}$ velocity, $\eta$ the effective viscosity with $\eta=m^{*} N / \tau$. As $V_{C D W}=\lambda_{C D W} v$, where $v$ is the frequency which is obtained from NBN measurements, the consequence of equation (3) would be a linear relation between the electric field and the frequency $v$, which is not experimentally shown in small electric field. In high electric field we have experimentally observed a saturation for $\mathrm{dE} / \mathrm{dv}$ which allows us to deduce $\tau^{-1}$ :

$$
\frac{1}{\tau}=\left.\frac{\mathrm{e}}{\lambda_{\mathrm{CDW}} \mathrm{m}^{*}} \frac{\mathrm{dE}}{\mathrm{dv}}\right|_{\infty}
$$

(The symbol $\infty$, for $\mathrm{dE} / \mathrm{dv}$, means that we have taken the saturated values obtained in our experimental electric field limit). Our results will be now analysed in the light of the theory for the ac response of the collective mode in CDW systems which has been elaborated by $W$. Wonneberger [14]. Extending earlier work of Littlewood [15], Wonneberger's theory is based on an extended Fukuyama-Lee-Rice (FLR) phase approach to the ac conductivity including quasi particle effects. One important advantage of this theory is that it provides a tractable analytical formula for $\sigma(\omega)$. In the weak pinning limit the formula for $\sigma(\omega)$ contains four frequencies: bare phason damping $\tau_{\mathrm{o}}{ }^{-1}$, longitudinal optical phason frequency $\omega_{\text {Lo }}$ with $\omega_{\mathrm{L}}=4 \pi \mathrm{Ne}^{2} / \mathrm{m}^{*} \varepsilon_{\Delta}$, dielectric relaxation frequency of quasi particles $\omega_{\mathrm{r}}$, with $\omega_{\mathrm{r}}=\left(4 \pi / \varepsilon_{\Delta}\right) \sigma_{\mathrm{qp}}$, and weak pinning frequency $\omega_{\mathrm{w}}$. The quantity $\varepsilon_{\Delta}$ is the dielectric constant from the Peierls gap, $\sigma_{\mathrm{qp}}$ is the quasi particle conductivity. Besides $\omega_{\mathrm{w}}$ they all can be inferred from experiments.

In the low frequency range or DC limit, Wonneberger has suggested [16] to take for $\tau^{-1}$ :

$$
\tau^{1}=\tau_{0}^{-1}+\left(\frac{1}{16 \pi} \frac{\omega_{W}}{\omega_{L_{0}}} \sqrt{\left.\frac{\varepsilon_{C D W}(0)}{\varepsilon_{\Delta}}\right)\left(\tau_{0}^{-1}+\frac{\omega_{L_{0}}^{2}}{\omega_{\tau}}\right.}\right)
$$

Using equation (4) for $\tau^{-1}$ equation (5) can be rewritten as :

$$
\left.\frac{\mathrm{dE}}{\mathrm{dv}}\right|_{\infty} \frac{\mathrm{e}}{\lambda_{\mathrm{CDW}}}=\frac{\mathrm{Ne}^{2}}{\sigma_{\mathrm{M}}}+\mathrm{B} \sqrt{\mathrm{m}^{*} \mathrm{Ne}^{2}}\left[\sigma_{\mathrm{M}^{-1}}+\sigma_{\mathrm{qp}}{ }^{-1}\right]
$$

where $B$ is a constant depending on $\omega_{w}$ and $\varepsilon_{C D W}(0)$

We make the hypothesis that, at least in a first approximation, in the right side of equation (6) $\sigma_{\mathrm{qp}}{ }^{-1}$ is the only term magnetic field dependent. In equation (6) we can introduce $\mathrm{dE} /\left.\mathrm{dv}\right|_{\infty}$ obtained in measurements without and with magnetic field $(7 \mathrm{~T})$, this enables us to calculate $B \sqrt{\mathrm{m}^{*}}$ and then $\sigma_{\mathrm{M}}$. 
To calculate $\tau_{\mathrm{o}}{ }^{-1}$ we must know $\mathrm{m}^{*}$. The order of magnitude has been given by Reagor et al. (17)Using more recent values for $\mathrm{N}_{\mathrm{T}}$ and $\mathrm{N}_{\mathrm{o}}\left(\mathrm{N}_{\mathrm{T}}=3.9 \times 10^{21}\right.$ carriers $/ \mathrm{cm}^{3}, \mathrm{~N}_{\mathrm{o}}=1.66 \times 10^{21}$ carriers $/ \mathrm{cm}^{3}$ ) [1], we have obtained $\mathrm{m}^{*} / \mathrm{m}_{\mathrm{e}}=63$.

We have assumed below $40 \mathrm{~K} \mathrm{~N} / \mathrm{m}^{*}=\mathrm{N}_{\mathrm{o}} / \mathrm{m}^{*}$ with $\mathrm{m}^{*} / \mathrm{m}_{\mathrm{e}} \simeq 60$, and have deduced the thermal variation of $\tau_{0}^{-1}$ (see Fig. 5). Above $30 \mathrm{~K}$ the thermal variation of $\tau_{0}{ }^{-1}$ could be in agreement with $\tau_{\mathrm{o}}{ }^{-1} \propto \mathrm{AT}$ deduced by Reagor et al. [17], but below $30 \mathrm{~K}$ we have deduced $\tau_{\mathrm{o}}^{-1} \propto \mathrm{A}_{1} \mathrm{~T}^{4.4}$. Up to now the only precise theoretical prediction for the thermal variation of the bare phason damping was the calculation of Takada, Wong and Holstein, TWH [18].

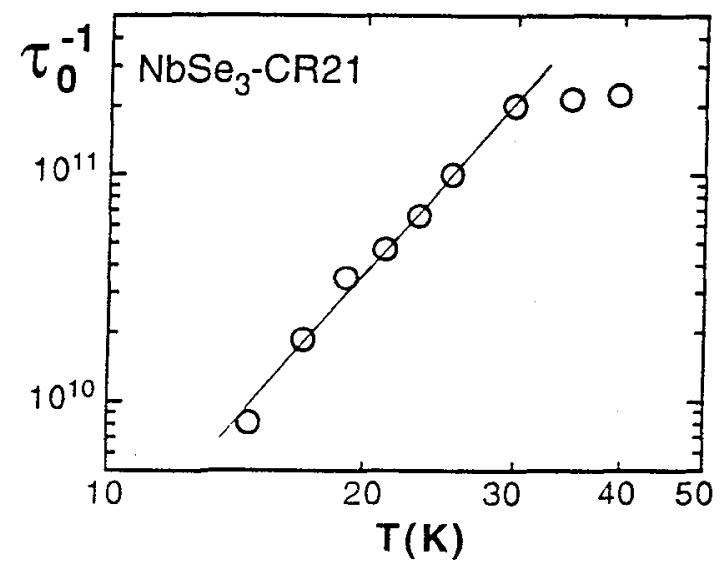

Figure 5 : Temperature dependence of the "bare" phason damping $\tau_{0}{ }^{-1}$. The solid line is a fit to $\tau_{0}{ }^{-1}=A_{1} T^{4.4}$.

According to these authors the dominant contribution arises from scattering with thermally ambient phasons. Damping due to impurities and normal electrons is neglected. In the low temperature regime $T<\theta_{D}\left(\theta_{D}:\right.$ Debye temperature, $\theta_{D} \simeq 100-150 \mathrm{~K}$ in $\left.\mathrm{NbSe}_{3}\right) \mathrm{TWH}$ have predicted :

$$
\tau_{\mathrm{o}}{ }^{-1}=\frac{16 \pi^{7}}{225 \mu} \frac{\lambda^{2}}{\Omega_{\mathrm{t}}^{4}} \mathrm{~T}^{5}
$$

where $\lambda$ is the electron-phonon-coupling constant, $\mu=\mathrm{m}^{*} / \mathrm{m}_{\mathrm{b}}$, with $\mathrm{m}_{\mathrm{b}}$ the band mass and $\Omega_{\mathrm{t}}$ is the transverse-phonon frequency. The $\mathrm{T}^{5}$ dependence for $\tau_{\mathrm{o}}{ }^{-1}$ is roughly observed in the present experiment. Regarding magnitude, using $\lambda=0.24, \mu=380$ and $\Omega_{\mathrm{t}}=35 \mathrm{~K}$ the theory yields $\tau_{0}-1=3 \times 10^{10} \mathrm{~Hz}$ at $\mathrm{T}=25 \mathrm{~K}$ a factor of 3 lower than the experimental results. Bearing in mind all the interlocking uncertainties concerning the basic parameters which enter into the theoretical expression for $\tau_{\mathrm{o}}{ }^{-1}$, a disagreement between theory and experiment by a factor of 3 is hardly surprising.

In conclusion of this study, we have shown that for the first time, from NBN measurements up to $400 \mathrm{MHz}$, we are able, first to measure the total damping constant $\tau^{-1}$, and then using the Wonneberger's theory to deduce the low temperature variation of the bare phason damping constant $\tau_{0}^{-1}$ which seems to verify the prediction of $T W H: \tau_{0}^{-1}=A_{1} T^{5}$.

Acknowledgements - The authors thank A. Bjelis, P. Monceau and M. Renard for helpful discussions. We are indebted to F. Lévy at Lausanne and R. Thorne at Cornell University for providing us with the samples. One of us (J.R) wishes to thank W. Wonneberger for illuminating discussions. 


\section{REFERENCES}

a) Permanent address : Institute of Radio-Engineering and Electronics Academy of Sciences of Russia.

[1] For a review see Electronic Properties of inorganic Quasi-One-Dimensional Compounds, edited by P. Monceau (Reidel, Boston, 1985).

[2] R.M. Fleming and C.C. Grimes, Phys. Rev. Lett. 42, 1423 (1979) and P. Monceau, J. Richard and M. Renard, Phys. Rev. Lett. 45, 43 (1980).

[3] J. Richard, P. Monceau and M. Renard, Phys. Rev. B 25, 948 (1982).

[4] J. Richard, J. Chen and S.N. Artemenko Solid State comm. 85, 605. (1993)

[5] H. Salva, Z.Z. Wang, P. Monceau, J. Richard and M. Renard, Philos. Mag. B 49, 385 (1984).

[6] Z.Z. Wang, P. Monceau, M. Renard, P. Gressier, L. Guemas, A. Meerschaut, Solid State Comm. 47, 439 (1983).

[7] S.E. Brown and G. Grüner, Phys. Rev. B 31, 8302 (1985).

[8] We have made new measurements of room temperature resistivity on different batches and the average value used to calculate $P$ is $200 \mu \Omega \times \mathrm{cm}$.

[9] M.L. Boriack and A.W. Overhauser, Phys. Rev. B 16, 5206 (1977) and P.A. Lee and T.M. Rice, Phys. Rev. B 9, 3870 (1979).

[10] S.N. Artemenko, E.N. Dolgov, A.N. Kruglov, Yu. I. Latyshev, Ya. S. Savitskaya and V.V. Frolov, Pisma ZhETF 39, 258 (1984).

[11] S.N. Artemenko, Synthetic. Metals 36, 381 (1990) and references therein.

[12] K. Maki and X. Huang, Phys. Rev. B 43, 5731 (1991).

[13] R.M. Fleming, R.J. Cava, L.F. Schneemeyer, E.A. Rietman, and R.G. Dunn, Phys. Rev. B 33, 5450 (1986).

[14] M. Bleher and W. Wonneberger, Solid State Commun. 69, 103 (1989);

T. Baier and W. Wonneberger, Z. Phys. B 79, 211 (1990);

W. Wonneberger, Synthetic Metals 43, 3793 (1991).

[15] P.B. Littlewood, Phys. Rev. B 36, 3108 (1987).

[16] Private communication. This limit can be derived from the calculation already published in ref. 14.

[17] D. Reagor, S. Sridhar and G. Grüner, Phys. Rev. B 34, 2212 (1986).

[18] S. Takada, K.Y.M. Wong and T. Holstein, Phys. Rev. B 32, 4639 (1985). 